\title{
A New Parameter for Product Configuration in Software Product Lines
}

\author{
Yuqing Lin, Huilin Ye, and Bojun Li \\ School of Electrical Engineering and Computer Science \\ The University of Newcastle, NSW2308, Australia. \\ e-mail: \{yuqing.lin, huilin.ye\}@newcastle.edu.au \\ bojun.li@studentmail.newcastle.edu.au
}

\begin{abstract}
Software product line development is a new software engineering method. It promotes the predictive software reuse by developing similar software systems together. One of the key artifacts of a software product line is the feature model, which represents the commonality and variability of all the products in the product family. When developing a member product from the product line, based on the requirement of the final product, features from the feature model will be selected and configured to form the final product. As the requirement could be incomplete or vague, the software engineer might need additional information in assisting with the feature selection. In this paper, we propose a new parameter, called the Instantiation Space, which measures the relative impact on the rest of the system for a selection. We believe that such information will be very helpful in the process of product configuration.
\end{abstract}

\section{Introduction}

Software product line engineering provides systematic approaches to exploit commonality and manage variabilities among a set of close related software products in a domain. It is well recognized in software industry that SPL based software development will achieve highlevel software reuse, improve the quality of the software development, reduce the cost, and improve software development productivity. There are two main processes in SPL based development, Domain Engineering and Application Engineering [2]. Domain Engineering is the process in which the commonality and the variability of the products in a domain line are identified and analyzed. Feature modelling is a well-known approach to modelling commonality and variability in software product lines. After examining existing products in a product family, a feature model is developed to abstract software system requirements or characteristics to features that both customers and developers can understand. In general, there are two types of features in software product lines, common features and variable features. Common features will be included in all member products, in other words, it is the commonality of all the products in a software product line. Variable features represent the variability among the products, i.e. different products will have different selections of the configurable variable features. The Application Engineering is the process in which products will be developed based on the results of domain engineering. The actual requirements of a certain product in a product line can be configured by using a feature model generated from domain engineering. When configuring a product based on a feature model all the common features will be included in the product and the variable features will be selected based on the constraints specified in the feature model.

A feature model usually uses a tree structure to organdie features at different levels. The variabilities of a product line are usually represented as it variation points in a feature model. In a feature tree, a variation point consists of a parent feature, a group of variable child features, called variants, and a multiplicity rule specifying the minimum and maximum number of variants that can be selected from the variation point when configuring a product. A valid selection will need to obey the multiplicity rule. The selection of variants at a variation point is not only constrained by the multiplicity but also by the dependencies between the variants at this variation point and the variants at other variation points. In this paper, we only consider two types of dependencies, "requires" and "excludes". These two types of dependency are used by most of the 
feature modelling approaches and are the major drivers for product configuration. If a variant A "requires" a variant $B$, then if variant $A$ is selected in a product, so will be the variant $B$. If a variant A "exclude" variant $B$ variant $A$ and $B$ can not be included in the same product.

The product configuration process is a decision making process, i.e. we will need to decide at each variation point that which feature should be included in the member product. Ideally, the requirement on the final product is complect and accurate. Then the whole configuration process is then straightforward. However, this is not the case in reality, it is hard to imagine that the requirement of the member product is complete and exact from beginning. Very often, the feature models drive requirement elicitation and analysis. Knowing which features are available in the software family may help customers decide which features their system should include. Because of the incompleteness of the requirements, the selection at the variation point can not be derived from the it. In this case, we will need to provide information to help customer to make selection. For example, we could let the customer understand what is the impact of the current selection to the rest of the system, or how much will the current selection restrict the future selections. In this paper, we would like to propose a parameter providing such type of information.

\section{A New Parameter for Member Prod- uct Configuration}

In the paper [1], authors have proposed a parameter called the variation degree. The variation degree reveals the instantiation space of the product line on basis of feature model. In other words, it tells how many different products can be derived from the feature model. This parameter reveals the complexity and flexibility of the product line. "It is of vital importance for deciding whether the PLFM captures the desired variability adequately" and "is necessary for planing the product line" [1]. The authors give a clear instruction of how this parameter is calculated when not considering the dependency relationships in the feature model. In the case that the dependency relationships is presented in the feature model, then authors only can give an upper and lower bound on value of this parameter.

In this paper, we would like to propose a new parameter. For a set of variants $V=\left\{v_{1}, v_{2}, . . v_{n}\right\}$, we define the Instantiation Space of $V$, denote by $I S(V)$ as the number of different products can be generated from the feature model if the set $V$ is included in the final product. This parameter has the similar idea as the parameter variation degree, however, this parameter is used in the product configuration. For all the variants associated with a variation point, we call a subset of variants a valid selection if it obeys the multiplicity. The complement of a valid selection is the rest of the variants which are not included in the selection. For a valid selection, it is important to know how much the selection restrict on the instantiation space. If a selection restricts the instantiation space of the feature model more than the other selections, it means that some features in the selection are having complex relationships with the rest of the feature model. Including these features in the final product will restrict very much the including of other features into the final product. Therefore, these features should be examined closely before select them to be included into the final product. If a selection do not restrict the instantiation space very much, it means the the feature has loose relationships with the rest of the feature model, and the selection does not restrict much on the future selections. In other words, the features included in the selections are not the key features. When the requirements of the final product are not complete, the initiation space of the selection give customer an idea that how the current selection affect the rest of the configuration and how much a selection restricts the final product.

To calculate this parameter for a valid selection, we will need to consider the dependency relationships the feature model. In [3], we have defined two measurements for a variant $v$, one is called the positive coverage $P C(v)$, another one is called the negative coverage $N C(v)$. The positive coverage is a set of variable features which we know will be included or excluded if the variant $v$ is included in the final product. The negative coverage is the set of variable features which we know will be included or excluded if the variant $v$ is excluded in the final product. For example, if variant $v$ requires variant $w$, then we know $w$ is in $P C(v)$, furthermore, if $w$ requires variant $u$, then $u$ is also in $P C(v)$ since if $v$ is included in the final product, then $u$ will be included as well. If variant $t$ requires variant $v$, then we know $t$ is in $N C(v)$, since if $v$ is not included in the final product, then $t$ can not be included as well. This simple logic calculation is straightforward to implement in a program.

Given a valid selection $s$ at the variation point $V P$, we will calculate the $I S(s)$ using a recursive function.

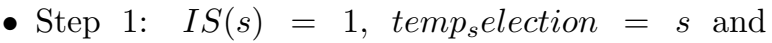
temp $p_{v} p=V P$

- Step 2: remove the variation point $t e m p_{v} p$ from 
the feature model.

- Step 3: calculate the positive coverage of temp selection and the negative coverage of the complement of temp - selection. Remove these two sets of variants from the feature model.

- Step 4: update the multiplicity of other variation points accordingly.

- Step 5: randomly select a variation point $V P_{x}$ and calculate the number of all valid selections $t$.

- Step 6: $I S(s)=I S(s) * t$ and temp-vp=VP

- Step 7: For each possible select $s_{x}$ at $V P_{x}$, tempselection $=s_{x}$ and repeat from Step 2 .

Below is an example, to illustrate how the parameter is calculated. The feature model presents a product family of vehicles. There are three variation points in the system, namely, Fuel Type, Transmission and Electronic Control. As illustrated in the figure, the variation point Fuel Type consists of three variants, Petrol, Diesel and Battery, the multiplicity specifies that there are up to two variants can be selected and included in the final product. Also there are exclude relationships between the the variant Petrol and Diesel. It means that there are 5 valid selections at this variation point, the fuel type of a vehicle could be using petrol, diesel, battery, hybrid petrol (battery + petrol), or hybrid Diesel (battery+diesel). The positive cover $P C($ Battery $)=$ \{Automatic, and Fuel Efficency Control\}, since based on the dependencies, including the Battery implies including the Automatic and Fuel Efficiency Control into the final product. To calculate IS(Battery), following the above algorithm, we first remove the variation point Fuel Type from the feature model, and we know the positive cover $P C($ Battery $)$ and the negative cover $N C($ Petrol, Diesel $)=\emptyset$. We will remove these two sets from the feature model as well. Next step is to update the multiplicity of other variation point. In this example, we will need to change the multiplicity at the variation point Transmission to 0 , which means that the dependencies has already implied the decision at this variation point and we do not need to consider this variation point anymore. Also, we will update the multiplicity of the Electronic Control to 0..2. There are totally 4 possible choices at the Electronic Control, so the $I S($ Battery $)=4$. Similarly, we get $I S($ Petrol $)=16$ and $I S($ Diesel $)=16$. From these values, we can see that the variant Battery has bigger impact to the rest of the system than other variants at the variation point Fuel Type, therefore, wether or not including Battery into the final product is one of the key decisions to make and need to be closely examined.

\section{Conclusions and Future Works}

In this paper, we have introduced an parameter which will measure how much the current selection restricts the instantiation space. the parameter gives a clear indication of how much impact the current selection has on the rest of the system. It will help user to make better selections in the product configuration process.

Randomly selecting a variation point, the sum of the initiation space of all possible valid selections give us the initiation space of the whole product line, which is the variation degree defined in [1]. Note that we have taken into account of the multiple dependencies in the calculation.

This parameter is also useful in various stages of software product line engineering. For example, if a given selection at a variation point having extremely too low instantiation space, that may indicate an inflexible product line, i.e. very limited number of products can be instantiated if the selection is made. Thus, we may need to look at the variants included in the selection to see if any improvement can be done for the feature model itself to make it more flexible to accommodate a larger number of product configuration. Also user could start a configuration from the variation point which including the selections of low instantiation space, this will help to save a lot of effort in the configuration. As the dependencies or constraints among the selections have been automatically satisfied the configuration process will be less error-prone. Due to the limitation of the space, we do not discuss the use of this parameter in detail in this paper.

In our approach, we have only considered two types of dependency relationships, the requires and excludes. In some of the feature modelling approaches, there are other types of relationships modelled and presented in the feature model, such as the impact relationships. Feature A impact on feature B could mean several things, for example, the selection of feature A suggest in certain degree of selection of feature $\mathrm{B}$, or the implementation of feature $\mathrm{A}$ is depend on the implementation of feature B etc. In this case, we will need to examine these complex relationships closely and define the proper logical operations to make our approach work. This will be our work in the near future.

Acknowledgement This work is supported by ARC project DP0772799. 


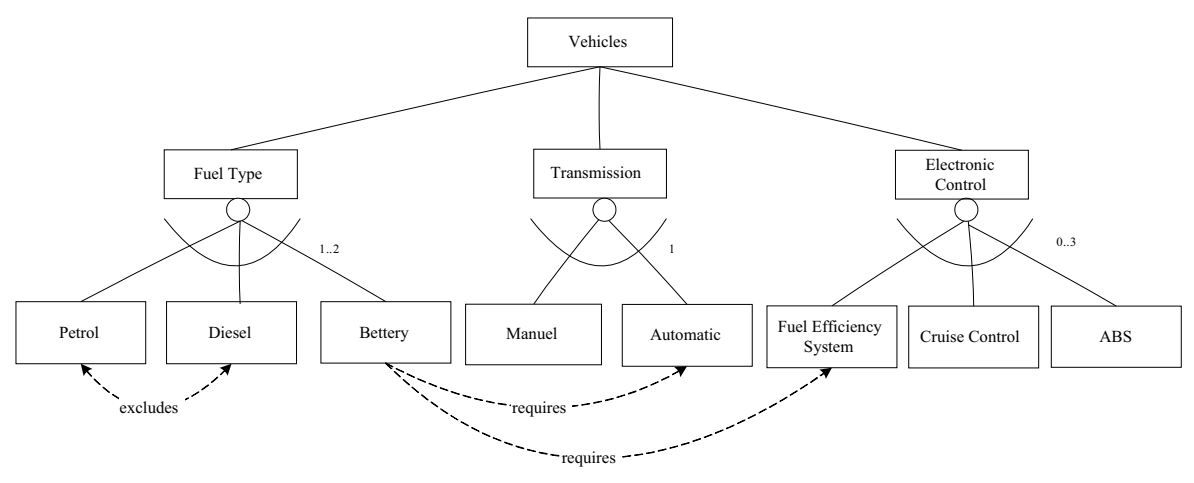

Figure 1. A feature model for Vehicles

\section{References}

[1] T. Maben and H. Lichter, Determining the variation Degree of feature models Software Product Line Conference, 2005. SPLC '05. 9th International (2005) 82-88.

[2] K. Pohl, G. Bockle, F. van der Linden, Software Product Line Engineering: Foundations, Principles and Techniques. Springer Berlin, ISBN: 9783540243724, 2005.

[3] Y. Lin, H. Ye, J. Tang, M. Murwantara, Mananging the Product Configuration in Software Product Line. Preprint. 